\title{
Effective methods to promote heat dissipation of wrist wearables
}

\author{
Kodai Matsuhashi ${ }^{1}$, Koutaro Hachiya ${ }^{2}$, Toshiki Kanamoto ${ }^{1}$, Shinsuke Kashiwazaki ${ }^{1}$, Kyosuke Kusumi ${ }^{1}$, \\ and Atsushi Kurokawa ${ }^{1, \text { a) }}$
}

\begin{abstract}
Wearable technology has been rapidly evolving. Many functions beyond current smartphone capabilities must be realized in wearables that are smaller than smartphones. Heat generation due to power consumption may cause both circuit malfunctions and low-temperature burns. This letter presents thermal design methods to promote heat dissipation of wrist wearables. First, the thermal model to easily obtain each part temperature is described. Next, belt heat dissipation effects are clarified by simulations with the model. The results indicate that each technique using the belt width, thickness, length, covering rubber, or heatsink has a high effect on heat dissipation. In addition, by combining these techniques, temperature rises of the display, bottom of device body, and belt can be reduced by $30.5 \%, 52.4 \%$, and $52.7 \%$.

Keywords: wearables, smart watches, thermal design, thermal management, thermal analysis

Classification: Devices, circuits and modules for IoT and biomedical applications
\end{abstract}

\section{Introduction}

With the advance of hardware and software technologies in compact electronic devices, the wearables market has grown dramatically over the last decade. The forecast from the International Data Corporation (IDC) Worldwide Quarterly Wearable Device Tracker was the 368.2 million in 2020, and it is expected to reach 526.8 million in 2024 [1]. Wrist wearables (e.g., smart watches, smart bands, smart bracelets, and fitness trackers), which are worn as small computers around the wrist, account for over half of the wearable devices in the market, and they are provided by vendors such as Apple, Xiaomi, and Fitbit [2].

Wrist wearable technologies have been researched in domains such as medicine and healthcare, sport, education, human-computer interaction, and security [3, 4, 5, 6, 7]. For medical uses, technologies have been presented for recognizing the user's behavior [8], Monitoring of the state of health $[9,10]$, remote medicine [11], medical emergency situations [12]. Wrist wearables use many sensors, including accelerometers, gyroscopes, magnetometers, pressure gauges, GPS, thermistors, and piezoelectric devices. Additionally, these sensors can measure numerous parameters, such as body movement, physical activity, temperature, and heart rate. Some technologies with sensors have been pre-

${ }^{1}$ Graduate School of Science and Technology, Hirosaki University, Aomori, Japan

${ }^{2}$ Graduate Program in Environmental Information Science, Teikyo Heisei University, Tokyo, Japan

a) kurokawa@hirosaki-u.ac.jp

DOI: $10.1587 /$ elex.18.20210017

Received January 12, 2021

Accepted February 3, 2021

Publicized February 10, 2021

Copyedited March 10, 2021 sented, such as finger-augmented RFID system [13] and intuitive teleoperation of robotic arm [14]. The touch interface of smart watches has been presented [15]. Moreover, technologies exploiting the temperature difference across a person's body to generate power by so-called "body-heat harvesting" have been proposed $[16,17]$. Technology to capture the motion of arm swing has also been proposed [18].

Wearable users expect such devices to have a long operational life in the manner of traditional watches. Under those circumstances, power consumption of most wrist wearables (like smart watches) is kept to less than $200 \mathrm{~mW}$ [19]. In contrast, power consumption of smart phones ranges from 0.5 to $3 \mathrm{~W}$ [20]. Thermal analysis and management of smart phones have been presented [20, 21, 22, 23]. Thermal management of smart watches has been reported [24]. To create future wrist wearables, it will be necessary to process more data at higher speed by using the state-of-the-art sensors and software technologies. In the near future, wrist wearables with functions and performance that exceed smart phones may be developed. Technologies for more efficiently charging those devices may also be developed. Further, scenarios in which such devices are used in the manner of high power consumption in a short time may increase. Thermal management for future wrist wearables will thus be essential. Wrist wearables users might wear the device for different periods of time: all day long (for daily health care such as patient monitoring); half a day or so (for only going out); or short times (such as during sports activities, driving, rehabilitation, and fitness training). The effect of the heating device with a wrist on physiology and temperature sensation has been researched [25]. Low-temperature burns may be caused if a device with operating temperature of $48^{\circ} \mathrm{C}$ is in contact with the skin for 10 minutes or if the device with that of $43^{\circ} \mathrm{C}$ is in contact with the skin for eight hours or longer [26]. We have presented thermal management methods for the future wrist wearable device [27]. In the paper, the maximum temperature in the ICs that generate heat was discussed. However, it hasn't analyzed most important surface temperatures for thermal designs of wearables, hasn't include a thermal model, and is not enough to present temperature reduction effects of the wearable belt.

In this letter, some important things for thermal design of wrist wearables are presented. We clarify each part temperature under various conditions. A difference between wrist wearables and other wearables is whether or not a belt is used. Belt heat dissipation effects for some techniques are clarified. In addition, it is shown that combinations of multiple techniques can greatly suppress the temperature rises at important parts. 
The rest of the letter is organized as follows. Section 2 describes the wrist wearable used in this work. Section 3 shows a thermal model for thermal simulation. Section 4 presents simulation results obtained using the thermal model. Section 5 concludes this letter.

\section{Wrist wearable}

A basic structure of a wrist wearable used in this work is shown in Fig. 1, where (a) is a top view and (b) is a side view. Under the basic conditions, device body size is $40 \times$ $40 \times 11 \mathrm{~mm}$

Fig. 2 shows the device body of the wrist wearable, which is composed of a display, 3D IC, package, PCB, battery, and case. Fig. 3 shows the package containing the 3D IC on the PCB. Size and thermal conductivity of the main parts are listed in Table I. Aluminum was used for the case and belt. Size and thermal conductivity of the 3D IC [28, 29] are listed in Table II. Thermal conductivity of the IC chips is expressed as equivalent values that account for the effect of through-silicon vias (TSVs).

\section{Thermal model}

\subsection{Thermal model of 3D IC}

For simplicity, it is assumed that the 3D IC depicted in Fig. 4 is used for the electronic device. One chip is composed of a

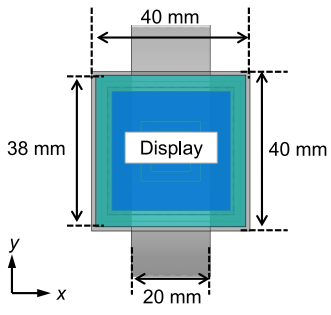

(a)

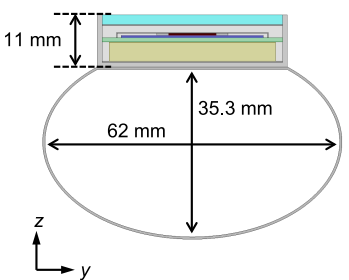

(b)
Fig. 1 Basic structure of wrist wearable used in this work: (a) top view and (b) side view.

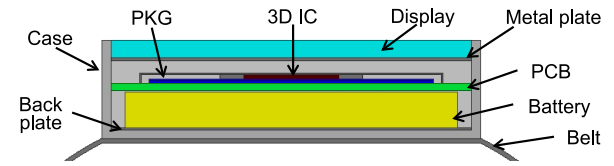

Fig. 2 Structures of device body of wrist wearable.

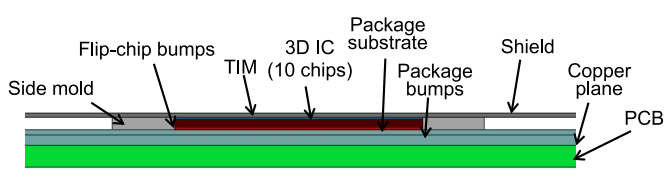

Fig. 3 Structures of package with IC.

Table I Thermal conductivity and size of main part.

\begin{tabular}{c|c|c}
\hline Parts & TC $(\mathrm{W} / \mathrm{mK})$ & Size $(\mathrm{mm})$ \\
\hline Display & $x=1.8, y=1.8, z=0.1$ & $x=38, y=38, z=2$ \\
Metal plate & 10 & $x=38, y=38, z=0.3$ \\
Battery & $x=15, y=15, z=10$ & $x=35, y=35, z=4$ \\
Back plate & 10 & $x=38, y=38, z=0.25$ \\
Case (Al) & 236 & $x=40, y=40, z=11$ \\
Belt (Al) & 236 & $l=167, w=20, t=0.5$ \\
\hline
\end{tabular}

metal layer, a device layer, and a chip substrate with TSVs. Micro-bumps are used for connecting the chips. Each layer of the 3D IC structure is modelled as an equivalent thermal resistance to be connected in series in the vertical direction.

Fig. 5 shows a simple thermal model for a 3D IC with multiple heat sources. This model reduces a thermal circuit shown in the form of a one-dimensional-heat-flow model for an $n$-chip stacked 3D IC (on the left of the figure) to a simple model (on the right of the figure). Thermal resistances of the chip substrate, device layer, metal layer, and micro-bumps are represented by $R_{C S}, R_{D L}, R_{M L}$, and $R_{M B}$, respectively. The simple model is reduced to two heat sources $\left(P_{C_{-} U}\right.$ and $\left.P_{C_{-} L}\right)$ and three thermal resistances $\left(R_{T}, R_{U}\right.$, and $\left.R_{L}\right)$. The power consumptions for upper and lower chips is represented by

$$
\begin{gathered}
P_{C_{-} L}=\frac{1}{\sum_{i=1}^{n-1} R_{i}} \times \sum_{i=1}^{n-1}\left(\left(\sum_{j=i}^{n-1} R_{j}\right) P_{C, i}\right), \\
P_{C_{-} U}=\sum_{i=1}^{n} P_{C, i}-P_{C_{-} L},
\end{gathered}
$$

where $R_{k}(k=1, \cdots, n-1)$ is expressed by

$$
\begin{aligned}
R_{k}= & R_{D L_{-} z, k}+R_{C S_{\_} z, k} \\
& +R_{M B_{-} z, k+1}+R_{M L_{-} z, k+1}+R_{D L_{-} z, k+1} .
\end{aligned}
$$

In the simple model shown in Fig. $5, R_{T}$ is total thermal resistance between two heat sources $\left(P_{C_{-} U}\right.$ and $\left.P_{C_{-} L}\right)$, which is calculated as

Table II Thermal conductivity and size of package (with 3D IC).

\begin{tabular}{c|c|c}
\hline Component & TC $(\mathrm{W} / \mathrm{mK})$ & Size $(\mathrm{mm})$ \\
\hline Metal layer & $x=98, y=98, z=110$ & $x=10, y=10, z=0.003$ \\
Device layer & $x=144, y=144, z=156$ & $x=10, y=10, z=0.001$ \\
Chip substrate & $x=144, y=144, z=156$ & $x=10, y=10, z=0.02$ \\
Micro-bumps & 50 & $x=10, y=10, z=0.005$ \\
Flip-chip bumps & 60 & $x=10, y=10, z=0.08$ \\
Shield & 15 & $x=32, y=32, z=0.15$ \\
Mold & 0.88 & $x=15, y=15, z=0.465$ \\
Package substrate & 149 & $x=30, y=30, z=0.2$ \\
Package bumps & 33 & $x=30, y=30, z=0.2$ \\
Copper plane & 401 & $x=38, y=38, z=0.03$ \\
PCB & 13 & $x=38, y=38, z=0.8$ \\
\hline
\end{tabular}

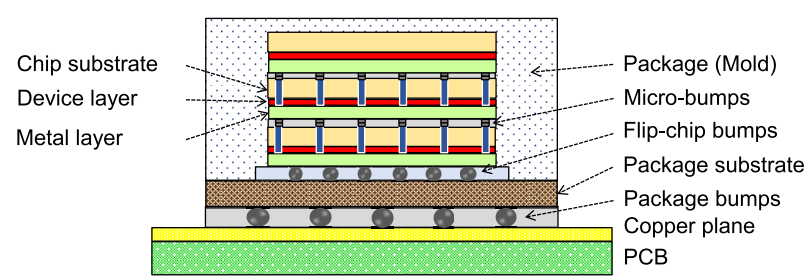

Fig. 4 Illustration of 3D IC structure.

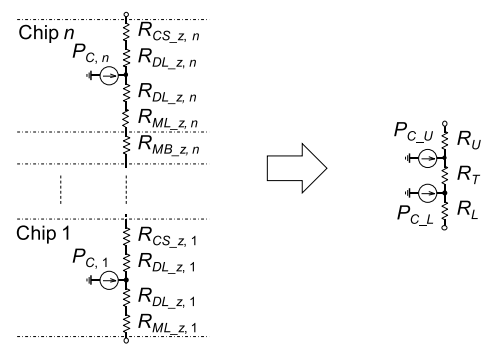

Fig. 5 Simple thermal model for 3D IC. 


$$
R_{T}=\sum_{i=1}^{n-1} R_{i}
$$

and upper and lower resistances $R_{U}$ and $R_{L}$ are expressed as

$$
\begin{aligned}
R_{U} & =R_{C S_{\_} z, n}+R_{D L_{-} z, n}, \\
R_{L} & =R_{D L_{-} z, 1}+R_{M L_{\_} z, 1} .
\end{aligned}
$$

\subsection{Whole model}

The whole thermal-circuit model for the wrist-worn wearable device is shown in Fig. 6. The total number of thermalresistance elements is only 83 . In the figure, $P_{D}$ represents power consumption (heat source) of the display.

\subsection{Verification}

The whole thermal model is verified as follows. The model was implemented in Microsoft Visual Basic. The temperature obtained by using the model in Fig. 6 and that obtained by using the commercial thermal solver [30] are compared in Fig. 7. In the figure, "node number" corresponds to the subscript number of the temperatures in Fig. 7. The results (temperatures) given by the model agree well with the results given by the solver. Maximum error in temperature rise to ambient temperature of $27^{\circ} \mathrm{C}$ was $2.5 \%$.

\subsection{Expected advantages}

We used the proposed thermal model for thermal simula-

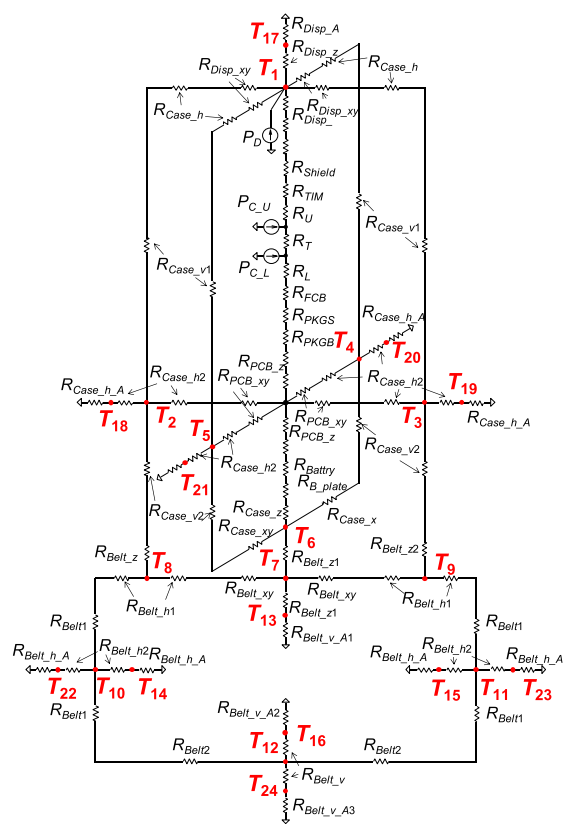

Fig. 6 Entire model for wrist wearable.

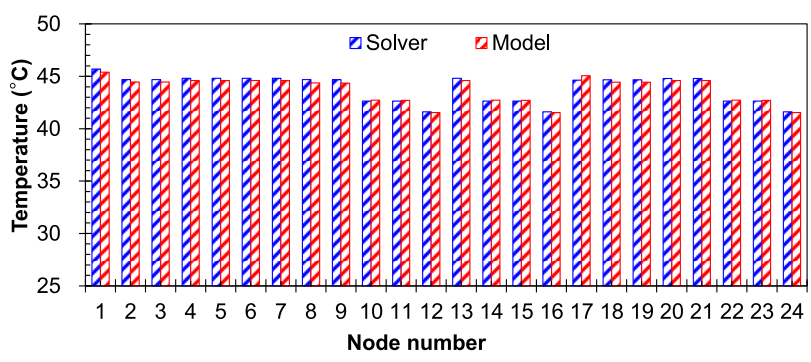

Fig. 7 Comparison of temperatures obtained by the model and solver. tion in this letter. The thermal model is consisted by a few thermal elements and is specialized in wrist wearables. By using the thermal model, temperatures at each part of the wrist wearable can be calculated at high speed. For instance, the calculation time taken by using the thermal circuit model was $0.32 \mathrm{sec}$, whereas that taken by using a thermal solver was $147 \mathrm{sec}$. The model is more than about 450 times faster. The model was executed by Intel Xeon CPU X5687 with 3.6 GHz. Moreover, the model can easily optimize characteristic parameters of each part. The designer can control physical structure properties by varying the thermal resistance values.

\section{Thermal simulation}

The basic conditions of the simulation are that the display's power consumption is $0.2 \mathrm{~W}$, the total power consumption (including that of the 3D IC) is $1 \mathrm{~W}$, the material, width, length, and thickness of the belt are aluminum, 20, 167, and $0.5 \mathrm{~mm}$, respectively, and the ambient temperature was $27^{\circ} \mathrm{C}$.

The measurement points of temperatures obtained by the thermal simulations are indicated in Fig. 8. "Display" indicates the position at the center of the top surface of the display, "Device" indicates the position on the back side of the belt under the device body, and "Belt" indicates the position on the back side of the belt, in the middle of the belt. The "Belt" and "Device" express the positions that touch the skin.

\subsection{Effects of belt size and materials}

Belt sizing in terms of width, thickness, and length is illustrated in Fig. 9. Aluminum (thermal conductivity of $236 \mathrm{~W} /(\mathrm{mK}))$ was used for the belt material.

The effect of heat dissipation from the belt is shown in Fig. 10. The effect of belt width is shown in Fig. 10(a). As the belt width increases, the heat dissipation effect becomes stronger. The belt-width effect is significant because the heat dissipation area expands. The effect of belt thickness is shown in Fig. 10(b). As belt thickness increases, temperatures at the simulation position of the display and device

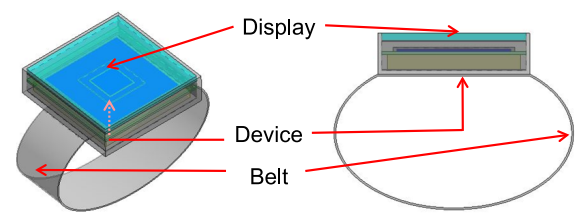

Fig. 8 Measurement points of temperatures obtained by thermal simulations.

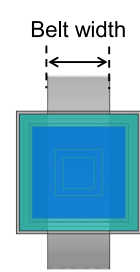

(a)

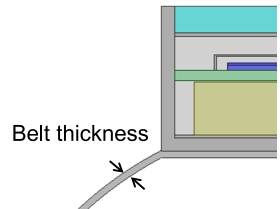

(b)

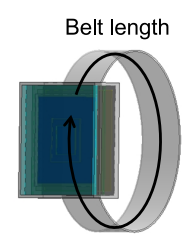

(c)
Fig. 9 Belt sizing: (a) width, (b) thickness, and (c) length. 


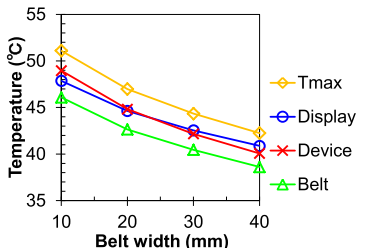

(a)

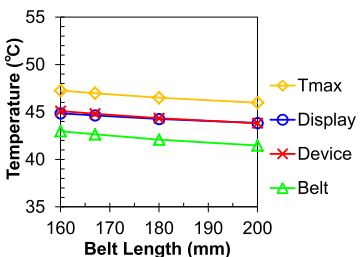

(c)

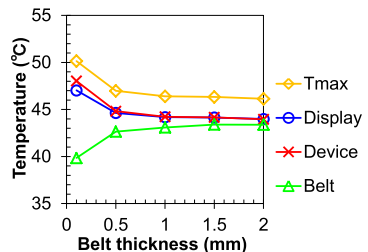

(b)

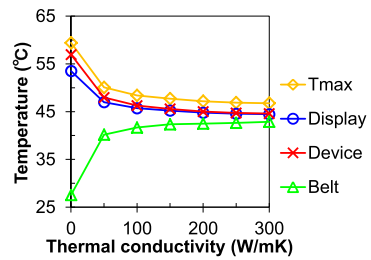

(d)
Fig. 10 Effect of heat dissipation from belt obtained by varying each parameters: (a) width, (b) thickness, (c) length, and (d) thermal conductivity.

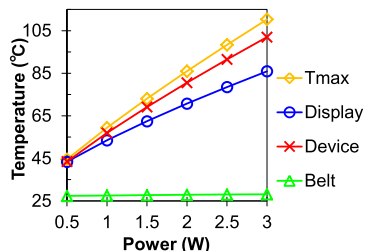

(a)

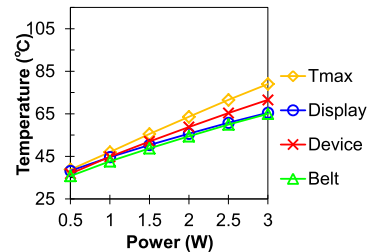

(b)
Fig. 11 Effect on temperatures at each part due to belt material: (a) rubber and (b) Al.

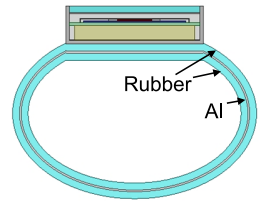

(a)

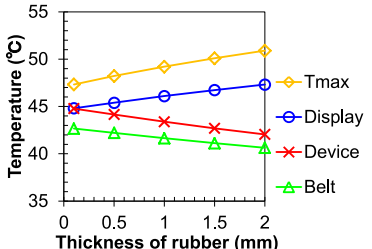

(b)
Fig. 12 Effect of thickness of rubber covering aluminum belt: (a) structure and (b) temperature.

decrease. However, the effect is saturated at around thickness of $1 \mathrm{~mm}$. Fig. 10(c) shows the effect of belt length. As belt length increases, temperature decreases. However, in the range of practical lengths, the effect is very small. The effect of thermal conductivity of the belt material is shown in Fig. 10(d). While thermal conductivity increases to $50 \mathrm{~W} /(\mathrm{mK})$, temperatures at the simulation positions of the display and device decrease significantly. However, when thermal conductivity exceeds about $100 \mathrm{~W} /(\mathrm{mK})$, temperature becomes nearly constant.

Difference in temperatures due to belt material (rubber or aluminum) when power consumption was varied is shown in Fig. 11. It is clear that the aluminum belt (which has higher thermal conductivity) is superior in terms of its heat dissipation effect.

\subsection{Effect of belt were covered with rubber}

Temperature characteristics in the case that both surfaces of the aluminum belt were covered with rubber are shown in Fig. 12. By increasing rubber thickness, skin-surface temperature is reduced. This method has the effect of reducing

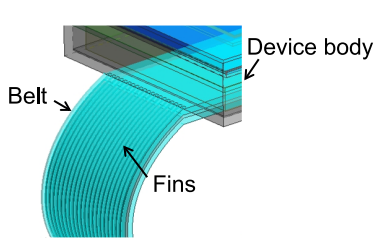

(a)

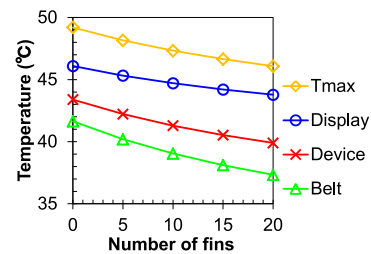

(b)
Fig. 13 Effect of belt with rubber fin heatsink: (a) structure and (b) temperature.

Table III Combination methods.

\begin{tabular}{c|c|c|c|c|c}
\hline Method & Width & Thickness & Length & $\begin{array}{c}\text { Covering } \\
\text { rubber }\end{array}$ & Heatsink \\
\hline M0 & --- & --- & -- & --- & --- \\
M1 & $\checkmark$ & $\checkmark$ & --- & --- & -- \\
M2 & --- & $\checkmark$ & $\checkmark$ & --- & -- \\
M3 & $\checkmark$ & --- & $\checkmark$ & --- & --- \\
M4 & $\checkmark$ & $\checkmark$ & $\checkmark$ & --- & -- \\
M5 & $\checkmark$ & $\checkmark$ & $\checkmark$ & $\checkmark$ & -- \\
M6 & $\checkmark$ & $\checkmark$ & $\checkmark$ & $\checkmark$ & $\checkmark$ \\
\hline
\end{tabular}

Table IV Temperature and reduction ratio of each method.

\begin{tabular}{c|c|c|c|c|c|c}
\hline \multirow{2}{*}{ Method } & \multicolumn{3}{|c|}{ Temperature $\left({ }^{\circ} \mathrm{C}\right)$} & \multicolumn{3}{c}{ Reduction Ratio (\%) } \\
\cline { 2 - 7 } & Display & Device & Belt & Display & Device & Belt \\
\hline M0 & 44.6 & 44.8 & 42.6 & 0 & 0 & 0 \\
M1 & 40.4 & 39.5 & 38.8 & 23.7 & 29.8 & 24.9 \\
M2 & 43.9 & 43.8 & 42.6 & 4.4 & 5.7 & 0.1 \\
M3 & 40.7 & 39.9 & 38.4 & 22.1 & 27.8 & 27.2 \\
M4 & 40.1 & 39.1 & 38.3 & 25.8 & 32.3 & 27.8 \\
M5 & 41.6 & 38.7 & 37.9 & 17.4 & 34.2 & 30.5 \\
M6 & 39.3 & 35.5 & 34.4 & 30.5 & 52.4 & 52.7 \\
\hline
\end{tabular}

temperatures of parts that always touch the skin but increasing the temperature of the display surface; in other words, it creates a trade-off relation.

\subsection{Effect of belt with fin heatsink}

Fin heatsink on the surface of the belt covered with rubber is shown in Fig. 13(a). The belt width of $20 \mathrm{~mm}$, rubber thickness of $1 \mathrm{~mm}$, and fin width of $0.5 \mathrm{~mm}$ were used. The effect of the belt with the fin heatsink on temperature is shown in Fig. 13(b). Due to the increase of the surface area of the belt, the heat-dissipation capability of the belt is grown. The temperature of "belt" in 20 fins and 0 fins are $41.6^{\circ} \mathrm{C}$ and $37.3^{\circ} \mathrm{C}$. It can reduce the temperature rise from ambient temperature by $29.4 \%$.

\subsection{Combinations of some techniques}

In this Section, we clarify the effects of combining the techniques shown in Sections 4.1 to 4.3 . We assume that the basic conditions are the belt width of $40 \mathrm{~mm}$, belt thickness of $1 \mathrm{~mm}$, belt length of $180 \mathrm{~mm}$, belt covered with rubber, and belt with fin heatsink, to the basic condition. Six combination methods are shown in Table III. Table IV shows results of the temperature and reduction ratio about temperature rise from ambient temperature of $27^{\circ} \mathrm{C}$ when M0 to M6 were used. The methods of M1 and M3-M6 can promote heat dissipation significantly. For example, M6 can reduce the temperature rise of "Display", "Device", and "Belt" by $30.5 \%, 52.4 \%$, and $52.7 \%$. 


\section{Conclusion}

In this letter, we presented effective methods for thermal design of wrist wearables. Thermal simulation results using a simple thermal model showed that physical and material parameters of the belt have great effects to promote heat dissipation of the wrist wearable. Moreover, the important part temperatures can be significantly reduced by using the combination of multiple techniques.

\section{References}

[1] International Data Corporation (IDC) Worldwide Quarterly Wearable Device Tracker: Worldwide wearables market braces for short-term impact before recovery in 2020 (2020) https://www.idc.com/.

[2] B2B Newz: Global wrist wearable market trend 2019 (2019) http://b2bnewz.com/.

[3] A. Kamišalić, et al.: "Sensors and functionalities of non-invasive wrist-wearable devices: a review," Sensors 18 (2018) 1714 (DOI: 10.3390/s18061714).

[4] R.M. Al-Eidan, et al.: "A review of wrist-worn wearable: sensors, models, and challenges," J. Sensors 2018 (2018) 5853917 (DOI: 10.1155/2018/5853917)

[5] F. De Arriba-Pérez, et al.: "Collection and processing of data from wrist wearable devices in heterogeneous and multiple-user scenarios," Sensors 16 (2016) 1538 (DOI: 10.3390/s16091538).

[6] Y.-L. Hsu, et al.: "Human daily and sport activity recognition using a wearable inertial sensor network," IEEE Access 6 (2018) 31715 (DOI: 10.1109/ACCESS.2018.2839766).

[7] G. Cosoli, et al.: "Wrist-worn and chest-strap wearable devices: systematic review on accuracy and metrological characteristics," Measurement 159 (2020) 107789 (DOI: 10.1016/j.measurement. 2020.107789).

[8] K. Ouchi, et al.: "LifeMinder: a wearable healthcare support system using user's context," 22nd International Conference on Distributed Computing Systems Workshops (2002) 791 (DOI: 10.1109/ICDCSW. 2002.1030864)

[9] F.P. Akbulut, et al:: "e-Vital: a wrist-worn wearable sensor device for measuring vital parameters," 2019 Medical Technologies Congress (2019) 1 (DOI: 10.1109/TIPTEKNO.2019.8894915)

[10] N. Sharma, et al.: "Fit-Wit: design and development of wearable healthcare device based on Intel Curie platform," 2017 International Conference on Computing, Communication and Automation (2017) 1271 (DOI: 10.1109/CCAA.2017.8230014).

[11] S. Kale, et al.: "Wearable biomedical parameter monitoring system: a review," 2017 International Conference of Electronics, Communication and Aerospace Technology (2017) 614 (DOI: 10.1109/ ICECA.2017.8203611).

[12] K. Wei, et al.: "Health monitoring based on internet of medical things: architecture, enabling technologies, and applications," IEEE Access 8 (2020) 27468 (DOI: 10.1109/ACCESS.2020.2971654).

[13] V. Di Cecco, et al.: "Finger-augmented RFID system to restore peripheral thermal feeling," 2017 IEEE International Conference on RFID (2017) 54 (DOI: 10.1109/RFID.2017.7945587).

[14] D. Gong, et al.: "Motion mapping from a human arm to a heterogeneous excavator-like robotic arm for intuitive teleoperation," 2019 IEEE International Conference on Real-time Computing and Robotics (2019) 493 (DOI: 10.1109/RCAR47638.2019.9044131).

[15] S.-C. Lim, et al.: "Expansion of smartwatch touch interface from touchscreen to around device interface using infrared line image sensors," Sensors 15 (2015) 16642 (DOI: 10.3390/s150716642).

[16] Y. Shi, et al.: "Wearable thermoelectric generator with copper foam as the heat sink for body heat harvesting," IEEE Access 6 (2018) 43602 (DOI: 10.1109/ACCESS.2018.2863018).

[17] V. Leonov: "Human machine and thermoelectric energy scavenging for wearable devices," ISRN Renewable Energy 2011 (2011) 785380 (DOI: 10.5402/2011/785380).

[18] M. Cai, et al.: "Self-powered smart watch and wristband enabled by embedded generator," Applied Energy 263 (2020) 114682 (DOI: 10.1016/j.apenergy.2020.114682).

[19] X. Liu, et al.: "Characterizing smartwatch usage in the wild," 15th Annual International Conference on Mobile Systems, Applications, and Services (2017) 385 (DOI: 10.1145/3081333.3081351).

[20] X. Chen, et al.: "Smartphone energy drain in the wild: analysis and implications," ACM SIGMETRICS Performance Evaluation Review (2015) 151 (DOI: 10.1145/2796314.2745875).

[21] J. Lee, et al.: "Parametric thermal modeling of heat transfer in handheld electronic devices," 2008 IEEE Intersociety Conference on Thermal and Thermomechanical Phenomena in Electronic Systems (2008) 604 (DOI: 10.1109/ITHERM.2008.4544324).

[22] Q. Xie, et al.: "Therminator: a thermal simulator for smartphones producing accurate chip and skin temperature maps," 2014 International Symposium on Low Power Electronics and Design (2014) 117 (DOI: 10.1145/2627369.2627641).

[23] Z. Luo, et al.: "System thermal analysis for mobile phone," Applied Thermal Engineering 28 (2008) 1889 (DOI: 10.1016/ j.applthermaleng.2007.11.025).

[24] A.Q. Han: "Thermal management and safety regulation of smart watches," 2016 IEEE Intersociety Conference on Thermal and Thermomechanical Phenomena in Electronic Systems (2016) 939 (DOI: 10.1109/ITHERM.2016.7517646).

[25] G. Lopez, et al.: "Development of a wrist-band type device for lowenergy consumption and personalized thermal comfort," 2016 11th France-Japan \& 9th Europe-Asia Congress on Mechatronics/17th International Conference on Research and Education in Mechatronics (2016) 209 (DOI: 10.1109/MECATRONICS.2016.7547143).

[26] A.P. Gagge, et al.: "An effective temperature scale based on a simple model of human physiological regulatory response," ASHRAE Trans. 77 (1971) 247.

[27] K. Matsuhashi and A. Kurokawa: "Thermal management for future wrist wearable devices," 2018 IEEE 3rd International Conference on Integrated Circuits and Microsystems (2018) 313 (DOI: 10.1109/ ICAM.2018.8596457).

[28] F. Tavakkoli, et al.: "Analysis of critical thermal issues in 3D integrated circuits," Int. J. Heat Mass Transfer 97 (2016) 337 (DOI: 10.1016/j.ijheatmasstransfer.2016.02.010).

[29] K. Furumi, et al.: "Cooling architectures using thermal sidewalls, interchip plates, and bottom plate for 3D ICs," 2017 International Symposium on Quality Electronic Design (2017) 283 (DOI: 10.1109/ ISQED.2017.7918329).

[30] Murata Software Co., Ltd.: Femtet ${ }^{\circledR} 2020.0$ (2020) http://www.muratasoftware.com/en. 